\title{
Dyslipidemia in Psoriasis: A Case - Controlled Study
}

\author{
Monique Kafle', Madhu Gyawalee', Amit Amatya', Bhaskar Mohan Meher Kayastha', \\ Smarika Upadhyaya²
}

\author{
${ }^{1}$ Patan Academy of Health Sciences (PAHS), Lalitpur, Nepal; ${ }^{2}$ Kathmandu University School of Medical Sciences
}

\begin{abstract}
Introduction: Psoriasis is a chronic inflammatory condition of skin which can be associated with other ailments including dyslipidemia, which is an abnormal serum lipid profile.

Objective: The aim of present study was to determine correlation between dyslipidemia and psoriasis of patients visiting the out-patient department of Patan Hospital

Methods: Ninety patients were selected for the study. Among them, 30 patients with psoriasis were taken as cases and 60 patients that matched for age and sex with psoriasis patients were taken as controls. The lipid profile that comprised four parameters: serum high-density lipoprotein, low-density lipoprotein, total cholesterol and triglyceride were determined and compared for both case and control groups.

Results: More than $95 \%$ of the psoriatic patients had dyslipidemia. The odds of dyslipidemia among psoriatic patients were 1.709 times higher compared to the control group $(p<0.001)$. Among the parameters, results showed highdensity lipoprotein was significantly lower while triglyceride was significantly higher among the cases compared to controls with $p$ value of 0.012 and 0.001 respectively. The risk factors like smoking and alcohol were present in higher proportions among the patients with dyslipidemia, but they failed to reach statistical significance. With increase in duration of psoriasis and PASI scores, lipid parameters i.e., cholesterol, triglyceride and density lipoprotein increased while high density lipoprotein decreased but this correlation was insignificant.

Conclusion: This study showed dyslipidemia is significantly higher among patients with psoriasis especially lipid profile parameters triglyceride and total cholesterol. Thus, timely screening and management of dyslipidemia among psoriatic patients might be helpful in prevention of future cardiovascular events.
\end{abstract}

Key words: Dyslipidemia; Lipid profile; Psoriasis

\section{Introduction}

P soriasis is a chronic inflammatory condition of the skin with an erythematous plaque and silvery white scales on the extensors, affecting almost every body area. WHO global report of 2016 showed a prevalence range of psoriasis between $0.09 \%$ and $11.4 \% .{ }^{1}$ The pathophysiology includes an increase in antigen presentation by dendritic cells to T-cell with resultant T-cell activation and secretion of type 1 (TH1) cytokines. ${ }^{2}$ Numerous researches in recent years have recognized psoriasis as a systemic disease with multi-

Funding: No

Conflict of Interest: No

Address of Correspondence

Dr. Monique Kafle

ORCID ID:000-0003-0555-0012

Lecturer, Patan Academy of Health Sciences (PAHS), Lalitpur, Nepal

Contact No: 9851203060

E-mail: moniquekafle@pahs.edu.np organ abnormalities and numerous complications. The risk of cardiovascular abnormalities, hypertension, dyslipidemia, atherosclerosis, diabetes mellitus type 2, obesity, chronic obstructive pulmonary disease, cerebral stroke, osteoporosis, cancer, and depression is higher among psoriatic patients. ${ }^{3}$

Dyslipidemia is a de-ranged or an abnormal serum lipid profile. ${ }^{4}$ Numerous studies show that psoriatic patients have a pro-atherogenic lipid profile with

Date of Submission: $23^{\text {rd }}$ July 2021

Date of Acceptance: $31^{\text {st }}$ August 2021

Date of Publication: $1^{\text {st }}$ October 2021

How to cite this article

Kafle M, Gyawalee M, Amatya A, Kayastha BMM, Upadhyaya S. Dyslipidemia in Psoriasis: A Case - Controlled Study. NJDVL 2021;19(2):39-43. https://doi.org/10.3126/njdvl.v19i2.38556.

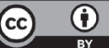

Licensed under CC BY 4.0 International License which permits use, distribution and reproduction in any medium, provided the original work is properly cited. 
raised levels of serum triglycerides (TAG), total cholesterol including low-density lipoprotein (LDL) and very low density lipoprotein (VLDL) cholesterol and lower levels of cardio protective high density lipoprotein(HDL) cholesterol. ${ }^{5}$ In Nepal one study by Poudyal et al. has reported serum TAG, total cholesterol (TC) levels significantly higher and serum HDL level significantly lower in patients with psoriasis. ${ }^{6}$

The aim of this study was to determine association between dyslipidemia and psoriasis among patients visiting dermatology OPD of Patan Hospital.

\section{Materials and Methods}

This study was designed as a case control study. The total number of patients were 90 , among them 30 were cases and 60 were controls i.e., allocation ratio of 1:2. The study subjects were taken from the dermatology OPD of Patan Hospital, Lalitpur. The period of study was six months from June 2020 to December 2020. Any patients presenting with chronic plaque type, pustular and guttate psoriasis were included as case; age and gender matched population of patients with skin ailments other than psoriasis were taken as control. The patients were diagnosed clinically along with disease severity of psoriasis assessed by PASI score. ${ }^{7}$

Patient with lipid lowering drugs, previously diagnosed cardiovascular disease before the onset of psoriasis, with diseases that can cause secondary hyperlipidemia before the onset of psoriasis such as hypothyroidism, diabetes mellitus, nephrotic syndrome, chronic renal insufficiency, obstructive liver disease, and connective tissue disease were excluded from the study.

A proforma was made for the case group as well as the control group. The questionnaire consisted of sociodemographic information, history of habits, history of psoriasis or skin ailments and lab findings of lipid profile.

All 30 cases were given adequate knowledge about dyslipidemia and investigation for lipid profile were only sent after a written consent from the patient. The control group that matched for age and sex with Psoriasis patients with no other systemic abnormalities were enrolled in the study. The data of the control group was taken from the Patan private clinic from patients and who came for a regular body checkup or needed medical requirements to travel abroad.

The biochemistry investigations for both both cases and controls from Patan Hospital were sent for lipid profile estimation. Detection of TC, HDL, and LDL was done using Vitros $4600^{\circledR}$ machine. All the patients with abnormal lipid profiles were referred to the medical referral clinic for further management of dyslipidemia.

The data was entered, edited and coded in Microsoft excel. The data were then exported to SPSS version 17.0 for further analysis. Comparison of dyslipidemia, smoking and alcohol between groups was done with the help of Chi square test. Odds ratios were calculated to estimate the risk. The mean lipid profile parameters between the groups were compared using independent $t$ test. Correlation between the duration and PASI scores with lipid profile parameters was tested with the help of Pearson's correlation coefficient. The level of significance was set at $5 \%$.

Informed written consent was taken from all study participants after explaining the study objectives with their right to withdraw from study any time they wanted. Anonymity and confidentiality of the study participants were assured and maintained. Prior approval of the study was obtained from the Institutional Review Committee, Patan academy of health science (PAHS).

\section{Results}

In this study, more than $95 \%$ of the psoriatic patients showed the presence of dyslipidemia, while among the controls, it was observed in about $60 \%$ of the patients. Comparison between the lipid profile parameters showed a higher proportion of psoriatic patients with deranged lipid profile findings compared to controls, as shown in Table 1.

Findings shows that a significant proportion of cases suffered from dyslipidemia compared to the controls with $p$ value $<0.001$. Also, the odds of psoriatic patients having dyslipidemia was 1.7 times higher compared to other non-psoriatic patients as shown in table 2.

The mean TC, TAG and LDL values are higher, while mean HDL values are lower among cases compared to controls. However, among the four parameters, it was found that HDL is significantly lower while TAG is significantly higher among the cases compared to controls with $p$ value of 0.012 and 0.001 respectively as shown in table 3 .

In the study, both smoking and alcohol were not significantly associated with dyslipidemia with $p$ value $>0.05$. Thus, smoking and alcohol are not associated with dyslipidemia, as shown in Table 4. 
In the study, a higher proportion of cases smoked tobacco while a slightly lower proportion of them consumed alcohol compared to the controls. However, both these findings failed to reach statistical significance highlighting that there is no association of tobacco and alcohol with psoriasis as shown in Table 5.

This study shows higher the PASI scores and longer the duration of psoriasis, the higher was the level of TC, TAG and LDL which is shown by positive correlation coefficient while there was a negative correlation with HDL scores. However, these correlations failed to reach statistical significance highlighting the lack of association between PASI scores and duration of psoriasis with various parameters of lipid profile as shown in table 6.

Table 1: Prevalence of dyslipidemia among cases and controls. $(n=90)$

\begin{tabular}{|l|c|c|}
\hline Prevalence & Cases $\mathbf{( n = 3 0 )}($ Count, $\%)$ & Controls $(\mathbf{n}=\mathbf{6 0})($ Count, $\%)$ \\
\hline Dyslipidemia & $29(96.7 \%)$ & $37(61.7 \%)$ \\
\hline Total Cholesterol $(>200 \mathrm{mg} / \mathrm{dl})$ & $10(33.3 \%)$ & $12(20.0 \%)$ \\
\hline Triglycerides $(>150 \mathrm{mg} / \mathrm{dl})$ & $20(66.7 \%)$ & $19(31.7 \%)$ \\
\hline High Density Lipoprotein $(<40 \mathrm{mg} / \mathrm{dl})$ & $15(50.0 \%)$ & $16(26.7 \%)$ \\
\hline Low Density Lipoprotein $(>100 \mathrm{mg} / \mathrm{dl})$ & $16(53.3 \%)$ & $23(38.3 \%)$ \\
\hline
\end{tabular}

Table 2: Association of dyslipidemia with psoriasis. $(n=90)$

\begin{tabular}{|c|c|c|c|c|c|c|}
\hline & \multirow{2}{*}{$\begin{array}{c}\text { Cases } \\
(n=30)\end{array}$} & \multirow{2}{*}{$\begin{array}{l}\text { Controls } \\
(n=60)\end{array}$} & \multirow{2}{*}{ Odds ratio } & \multicolumn{2}{|c|}{$95 \% \mathrm{Cl}$ of OR } & \multirow{2}{*}{$P$ value } \\
\hline & & & & Lower & Upper & \\
\hline Dyslipidemia (+) & $29(96.7 \%)$ & $37(61.7 \%)$ & \multirow{2}{*}{1.709} & \multirow{2}{*}{1.359} & \multirow{2}{*}{2.150} & \multirow{2}{*}{$<0.001^{*}$} \\
\hline Dyslipidemia (-) & $01(03.3 \%)$ & $23(38.3 \%)$ & & & & \\
\hline
\end{tabular}

*Statistically significant.

Table 3: Association of different parameters of lipid profile between cases and controls. $(n=90)$

\begin{tabular}{|l|c|c|c|c|}
\hline Parameters & $\begin{array}{c}\text { Cases }(\mathbf{n}=\mathbf{3 0}) \\
(\text { Mean } \pm \mathbf{~ s d )}\end{array}$ & $\begin{array}{c}\text { Controls }(\mathbf{n}=6 \mathbf{0}) \\
(\text { Mean } \pm \mathbf{~ s d ) ~}\end{array}$ & Mean Difference & P value \\
\hline Total cholesterol & $185.2 \pm 34.7$ & $172.1 \pm 38.5$ & 13.10 & 0.120 \\
\hline Triglycerides & $209.5 \pm 104.0$ & $131.3 \pm 70.03$ & 78.15 & $0.001^{*}$ \\
\hline High density lipoprotein & $42.2 \pm 15.1$ & $51.4 \pm 16.5$ & -9.16 & $0.012^{*}$ \\
\hline Low density lipoprotein & $100.2 \pm 34.4$ & $94.4 \pm 27.2$ & 5.78 & 0.426 \\
\hline
\end{tabular}

*Statistically significant.

Table 4: Association of smoking and alcohol with dyslipidemia among study participants. $(n=90)$

\begin{tabular}{|c|c|c|c|c|c|c|}
\hline & \multirow{2}{*}{$\begin{array}{l}\text { Dyslipidemia (+) } \\
\qquad(n=66)\end{array}$} & \multirow{2}{*}{$\begin{array}{l}\text { Dyslipidemia (-) } \\
\qquad(n=24)\end{array}$} & \multirow{2}{*}{$\begin{array}{l}\text { Odds } \\
\text { ratio }\end{array}$} & \multicolumn{2}{|c|}{$95 \% \mathrm{Cl}$ of OR } & \multirow{2}{*}{$\begin{array}{c}P \\
\text { value }\end{array}$} \\
\hline & & & & Lower & Upper & \\
\hline Smoking (+) & 20 (30.3 \%) & 03 (12.5 \%) & \multirow{2}{*}{3.043} & \multirow{2}{*}{0.814} & \multirow{2}{*}{11.378} & \multirow{2}{*}{0.087} \\
\hline Smoking (-) & 46 (69.7 \%) & 21 (87.5 \%) & & & & \\
\hline Alcohol (+) & 25 (37.9 \%) & $05(20.8 \%)$ & \multirow{2}{*}{2.317} & \multirow{2}{*}{0.769} & \multirow{2}{*}{6.986} & \multirow{2}{*}{0.129} \\
\hline Alcohol (-) & 41 (62.1\%) & 24 (34.3 \%) & & & & \\
\hline
\end{tabular}

Table 5: Association of smoking and alcohol with Psoriasis among study participants. $(n=90)$

\begin{tabular}{|c|c|c|c|c|c|c|}
\hline & \multirow{2}{*}{$\begin{array}{c}\text { Cases } \\
(n=31)\end{array}$} & \multirow{2}{*}{$\begin{array}{l}\text { Controls } \\
(n=70)\end{array}$} & \multirow{2}{*}{ Odds ratio } & \multicolumn{2}{|c|}{$95 \% \mathrm{Cl}$ of $\mathrm{OR}$} & \multirow{2}{*}{$P$ value } \\
\hline & & & & Lower & Upper & \\
\hline Smoking (+) & $08(26.7 \%)$ & $15(25.0 \%)$ & \multirow{2}{*}{0.917} & \multirow{2}{*}{0.338} & \multirow{2}{*}{2.487} & \multirow{2}{*}{0.864} \\
\hline Smoking (-) & $22(73.3 \%)$ & 45 (75.0\%) & & & & \\
\hline Alcohol (+) & 09 (30.0 \%) & $21(35.0 \%)$ & \multirow{2}{*}{1.256} & \multirow{2}{*}{0.489} & \multirow{2}{*}{3.229} & \multirow{2}{*}{0.635} \\
\hline Alcohol (-) & 21 (70.0 \%) & 39 (65.0\%) & & & & \\
\hline
\end{tabular}


Table 6: Correlation of PASI score and duration of psoriasis with parameters of lipid profile. $(n=30)$

\begin{tabular}{|c|c|c|c|}
\hline Psoriasis & Parameters of lipid profile & Correlation coefficient $(\boldsymbol{r})$ & P value \\
\hline \multirow{3}{*}{$\begin{array}{c}\text { Psoriasis Area and Severity } \\
\text { Index scores }\end{array}$} & TC & +0.101 & 0.596 \\
\cline { 2 - 4 } & TAG & +0.205 & 0.278 \\
\cline { 2 - 4 } & HDL & -0.069 & 0.716 \\
\cline { 2 - 4 } & LDL & +0.01 & 0.955 \\
\hline \multirow{3}{*}{ Duration in months } & TC & +0.036 & 0.850 \\
\cline { 2 - 4 } & TAG & +0.128 & 0.502 \\
\cline { 2 - 4 } & HDL & -0.078 & 0.684 \\
\hline
\end{tabular}

\section{Discussion}

The relation between psoriasis and dyslipidemia was established in $1958 .^{8}$ Since then, many studies have been published. A study from Sweden showed that more than $95 \%$ of psoriatic patients associated with dyslipidemia showing HDL to be lower while TAG significantly higher, ${ }^{9}$ which was a similar finding from Brazil, ${ }^{10}$ Japan, ${ }^{11}$ and Isreal. ${ }^{12}$ Causes of dyslipidemia in psoriasis may be multiple which including immune mechanisms involving IL-6 and tumor necrosis factor, C-reactive protein, and cellular oxidative stress. ${ }^{13}$ These factors alter the lipid metabolism. Similarly some studies suggest cytokines secreted by Th1 and Th17 may increase serum lipid levels with increased hepatic de novo fatty acid synthesis and hepatic lipid secretion. ${ }^{14,15}$

In a study performed in western China ${ }^{16}$ it has been found out that dyslipidemia is associated with smoking cigarette but in contrary to that our study has failed to show such a relation. Similarly, a study identifies a significant relation of alcohol intake with dyslipidemia ${ }^{17}$ but our study shows the contrary report to these findings. These two studies were performed independently without taking psoriasis into account.

Our study showed there is no relationship between smoking and alcohol use in psoriasis. A study shows alcohol use as an essential risk factor, particularly in male psoriasis patients. ${ }^{18}$ The same study gave the reason that there is an increased chance of psoriasis because alcohol use leads to an increase in trauma or infection. Another study suggested that the effect of ethanol on lymphocyte transformation leading to immune mechanisms which in turn increase the chance of psoriasis. ${ }^{19}$ A study by Fortes et. al. finds that severe psoriasis risk increased $30 \%$ in smokers. ${ }^{20}$ In contrary to these two studies, study conducted by Mallbris et al. ${ }^{9}$ on 200 patients found that factors like smoking and high alcohol consumption were not likely explanatory for lipid abnormalities which is similar to our findings.

In our study, there was an increase in the serum total cholesterol levels and LDL, TAG, TC; however there was a decrease in $\mathrm{HDL}$ in patients with long term psoriasis (more than 5 years) as mentioned in table 3 . Though the changes in HDL based on disease severity and duration were not significant, they formed an interesting observation. HDL levels, which are protective against cardiovascular risk, showed changes different from other lipids. This could signify increased risk and systemic damage with disease severity and duration.

Till now knowledge is derived from studying psoriasis patients without considering disease duration. Mallbris et al. ${ }^{9}$ showed the lipid changes at the onset of the disease. According to our study, an increasing trend in the lipids was observed with disease duration.

PASI score was used to grade the cases into mild, moderate, and severe. This score is considered as outstanding when severe cases are involved. It also provides the advantage of a large base of studies in which it has been used for comparison. ${ }^{21}$ In our study there was no significant correlation of dyslipidemia with disease severity based on PASI; however, we observed a fall in the levels of protective HDL in severe psoriasis as compared to mild cases.

The limitation of this study highlights a small sample size with a short time frame therefore fluctuating trends of lipids could not be evaluated for a longer period. Long term, multicentric study with larger sample size may be necessary to draw a definite conclusion.

\section{Conclusion}

This study shows relation between dyslipidemia and psoriasis. Whether or not the role of psoriasis in cardiovascular disease is yet to be studied along with that the relation between severity of psoriasis and dyslipidemia is to be established. Based on the current findings, we recommend that timely screening protocol for estimation of lipid profile among psoriatic patients can be initiated with goal to prevention, diagnosis and management of dyslipidemia as an established risk factor for cardiovascular diseases. 


\section{References}

1. Michalek IM, Loring B, John SM. Global report on psoriasis: World Health Organization; 2016.

2. Späh F. Inflammation in atherosclerosis and psoriasis: common pathogenic mechanisms and the potential for an integrated treatment approach. Br J Dermatol. 2008;159:10-7. https:// doi.org/10.1111/j.1365-2133.2008.08780.x

3. Nijsten T, Wakkee M. Complexity of the association between psoriasis and comorbidities. J Invest Dermatol. 2009;129(7):1601-3. https:// doi.org/10.1038/jid.2009.55

4. Pietrzak A, Michalak-Stoma A, Chodorowska G, Szepietowski JC. Lipid disturbances in psoriasis: an update. Mediators Inflamm. 2010;2010:535612. https://doi.org/10.1155/2010/535612

5. Javidi Z, Meibodi NT, Nahidi Y. Serum lipids abnormalities and psoriasis. Indian J Dermatol. 2007;52(2):89-92. https://doi.org/10.4103/00195154.33285

6. Poudyal Y, Rajbhandari S. Lipid profile in psoriasis. Journal of Universal College of Medical Sciences. 2014;2(1):16-9. https://doi.org/10.3126/jucms. v2i1.10485

7. Okun MM. Psoriasis Area and Severity Index: nuts and bolts of measuring disease severity in psoriasis. Clinics in dermatology. 2008;26(6):653-6. https://doi.org/ 10.1016/j. clindermatol.2008.08.002

8. Lea WA Jr, Cornish HH, Block WD. Studies on serum lipids, proteins, and lipoproteins in psoriasis. J Invest Dermatol. 1958;30(4):181-5

9. Mallbris L, Granath F, Hamsten A, Ståhle M. Psoriasis is associated with lipid abnormalities at the onset of skin disease. J Am Acad Dermatol. 2006;54(4):614-21. https://doi.org/10.1016/j. jaad.2005.11.1079

10. Rosa DJ, Machado RF, Matias FA, Cedrim SD, Noronha FL, Gaburri D, et al. Influence of severity of the cutaneous manifestations and age on the prevalence of several cardiovascular risk factors in patients with psoriasis. J Eur Acad Dermatol Venereol. 2012;26(3):348-53. https://doi. org/10.1111/j.1468-3083.2011.04076.x

11. Takahashi $H$, lizuka H. Psoriasis and metabolic syndrome. J Dermatol. 2012;39(3):212-8. https:// doi.org/10.1111/j.1346-8138.2011.01408.x

12. Dreiher J, Weitzman D, Davidovici B, Shapiro J, Cohen AD. Psoriasis and dyslipidaemia: a population-based study. Acta Derm Venereol. 2008;88(6):561-5. https://doi. org/10.2340/00015555-0510

13. Asha K, Singal A, Sharma SB, Arora VK, Aggarwal A. Dyslipidaemia \& oxidative stress in patients of psoriasis: Emerging cardiovascular risk factors. Indian J Med Res. 2017;146(6):708-13. https:// doi.org/10.4103/ijmr.IJMR_717_16

14. Feingold KR, Grunfeld C. Role of cytokines in inducing hyperlipidemia. Diabetes. 1992;41:97101. https://doi.org/10.1161/01.atv.14.11.1866

15. Memon RA, Grunfeld C, Moser AH, Feingold KR. Tumor necrosis factor mediates the effects of endotoxin on cholesterol and triglyceride metabolism in mice. Endocrinology. 1993;132(5):2246-53. https://doi.org/10.1210/ endo.132.5.8477669

16. Tan XJ, Jiao GP, Ren YJ, Gao XR, Ding $Y$, Wang $X R$, et al. Relationship between smoking and dyslipidemia in western Chinese elderly males. J Clin Lab Anal. 2008;22(3):159-63. https://doi. org/10.1002/jcla.20235

17. Shen Z, Munker S, Wang C, Xu L, Ye H, Chen $\mathrm{H}$, et al. Association between alcohol intake, overweight, and serum lipid levels and the risk analysis associated with the development of dyslipidemia. J Clin Lipidol. 2014;8(3):273-8. https://doi.org/10.1016/j.jacl.2014.02.003

18. Zhang $\mathrm{X}$, Wang $\mathrm{H}$, Te-Shao $\mathrm{H}$, Yang $\mathrm{S}$, Wang $F$. Frequent use of tobacco and alcohol in Chinese psoriasis patients. Int J Dermatol. 2002;41(10):659-62. https://doi.org/10.1046/ j.1365-4362.2002.01595.x

19. Naldi L, Peli L, Parazzini F. Association of early-stage psoriasis with smoking and male alcohol consumption: evidence from an Italian case-control study. Arch Dermatol. 1999;135(12):1479-84. https://doi.org/10.1001/ archderm.135.12.1479

20. Fortes C, Mastroeni S, Leffondre K, Sampogna F, Melchi F, Mazzotti $E$, et al. Relationship between smoking and the clinical severity of psoriasis. Arch Dermatol. 2005;141(12):1580-4. https:// doi.org/10.1001/archderm.141.12.1580

21. Spuls PI, Lecluse LL, Poulsen M-LN, Bos JD, Stern RS, Nijsten T. How good are clinical severity and outcome measures for psoriasis?: quantitative evaluation in a systematic review. J Invest Dermatol. 2010;130(4):933-43. https://doi. org/10.1038/jid.2009.391 